\title{
Switchgrass (Panicum virgatum L.) polyubiquitin gene (PvUbi1 and PvUbi2) promoters for use in plant transformation
}

David GJ Mann ${ }^{1,4^{*}}$, Zachary R King ${ }^{2,4}$, Wusheng Liu', Blake L Joyce', Ryan J Percifield ${ }^{3,4}$, Jennifer S Hawkins ${ }^{3,4}$, Peter R LaFayette ${ }^{2,4}$, Barbara J Artelt ${ }^{2,4}$, Jason N Burris ${ }^{1,4}$, Mitra Mazarei ${ }^{1,4}$, Jeffrey L Bennetzen ${ }^{3,4}$, Wayne A Parrott ${ }^{2,4}$ and Charles N Stewart Jr ${ }^{1,4}$

\begin{abstract}
Background: The ubiquitin protein is present in all eukaryotic cells and promoters from ubiquitin genes are good candidates to regulate the constitutive expression of transgenes in plants. Therefore, two switchgrass (Panicum virgatum L.) ubiquitin genes (PvUbi1 and PvUbi2) were cloned and characterized. Reporter constructs were produced containing the isolated $5^{\prime}$ upstream regulatory regions of the coding sequences (i.e. PvUbi1 and PvUbi2 promoters) fused to the uidA coding region (GUS) and tested for transient and stable expression in a variety of plant species and tissues.

Results: PVUbi1 consists of 607 bp containing cis-acting regulatory elements, a 5' untranslated region (UTR) containing a $93 \mathrm{bp}$ non-coding exon and a $1291 \mathrm{bp}$ intron, and a $918 \mathrm{bp}$ open reading frame (ORF) that encodes four tandem, head -to-tail ubiquitin monomer repeats followed by a 191 bp 3' UTR. PVUbi2 consists of 692 bp containing cis-acting regulatory elements, a 5' UTR containing a 97 bp non-coding exon and a 1072 bp intron, a 1146 bp ORF that encodes five tandem ubiquitin monomer repeats and a 183 bp 3' UTR. PVUbil and PVUbi2 were expressed in all examined switchgrass tissues as measured by qRT-PCR. Using biolistic bombardment, PvUbi1 and PvUbi2 promoters showed strong expression in switchgrass and rice callus, equaling or surpassing the expression levels of the CaMV 35S, 2x35S, ZmUbi1, and OsAct1 promoters. GUS staining following stable transformation in rice demonstrated that the PVUbi1 and PVUbi2 promoters drove expression in all examined tissues. When stably transformed into tobacco (Nicotiana tabacum), the PvUbi2+3 and PvUbi2+9 promoter fusion variants showed expression in vascular and reproductive tissues.
\end{abstract}

Conclusions: The PvUbi1 and PvUbi2 promoters drive expression in switchgrass, rice and tobacco and are strong constitutive promoter candidates that will be useful in genetic transformation of monocots and dicots.

\section{Background}

Genetic transformation is an important tool for crop improvement and research in genetics. The transformation of bioenergy crops with genes that alter plant development rate, growth habit, cell wall structure and/or composition has been deemed a promising approach to reduce cell wall recalcitrance (i.e., resistance to enzymatic degradation during saccharification) or to increase biomass yields [1-4]. Switchgrass (Panicum virgatum L.), a C4 perennial grass

\footnotetext{
* Correspondence: dmann1@utk.edu

'Department of Plant Sciences, University of Tennessee, Knoxville, TN 37996, USA

Full list of author information is available at the end of the article
}

species native to the prairies of North America, is a candidate lignocellulosic feedstock for bioenergy [5-7]. The development of tissue culture and transformation systems has led to significant breakthroughs and applications in switchgrass biotechnology [8-11]. Recent increases in transformation efficiency [12], along with recent demonstrations of transgenic modifications [13-15], suggest that genetic improvements of switchgrass through transgene expression and down-regulation of native genes will be accomplished with increasing regularity in the coming years.

Transformation of switchgrass and other monocots is facilitated by reliable molecular tools, including improved

\section{Biomed Central}


promoters for transgene expression. A variety of promoters used in monocot plant species have been reported in the literature, such as the rice (Oryza sativa) actin 1 (OsAct1) and actin 2 (OsAct2) promoters [16,17], the maize ubiquitin 1 (ZmUbi1) promoter [18], and multiple rice ubiquitin ( $R U B Q 1, R U B Q 2$, rubi3) promoters $[19,20]$. However, relatively few promoters have been used in the production of transgenic switchgrass. Richards et al. [9] demonstrated that the OsAct1and ZmUbi1 promoters are able to drive transgene expression in switchgrass, and these promoters have been used in subsequent switchgrass transformation studies [11,21,22]. While the cauliflower mosaic virus (CaMV) $35 \mathrm{~S}$ promoter has been used in switchgrass, it typically resulted in lower levels of expression $[21,23,24]$. Somleva et al. [10] used the rice ubiquitin 2 (rubi2) promoter with limited success, whereas the $c a b-m 5$ light-inducible promoter from the chlorophyll $a / b$-binding protein in maize fused to the heat shock protein 70 ( $h s p 70)$ intron resulted in significantly higher levels of transgene expression. However, $c a b-m 5$ expression is only expressed in a limited number of plant tissues and cell types [10,25,26]. Most recently, the rubi3 promoter was used to drive $s G F P$ expression in switchgrass callus, stem, axillary bud and anther tissues [12,27]. Discovery and characterization of new promoters with enhanced levels of constitutive expression are needed [28], and would be highly beneficial to improve bioenergy feedstock crops through genetic transformation. While tissue-specific and inducible promoters are desirable for certain applications $[29,30]$, constitutively expressed promoters are still the most commonly used promoters in transgenic plants and are advantageous for their wide range and stable levels of transgene expression.

Ubiquitin is a protein that consists of tandem repeats of a 76 amino acid monomer and is among the most conserved proteins in eukaryotes; only three amino acid polymorphisms exist among sequences from higher plant and animal species [31]. Ubiquitin is present in all eukaryotic cells. Therefore, the promoters from polyubiquitin genes are good candidates to regulate the constitutive expression of transgenes. Polyubiquitin gene promoters have been isolated from a variety of plants and tested for their ability to drive transgene expression, including from Arabidopsis thaliana [32], sunflower (Helianthus annuus) [33], parsley (Petroselinum crispum) [34], tobacco (Nicotiana tabacum) [35], potato (Solanum tuberosum) [36,37], tomato (Solanum lycopersicum) [38,39], sugarcane (Saccharum oficinarum) [40], Gladiolus sp. [41], soybean (Glycine max) [42] and Lotus japonicus [43] as well as the rice and maize polyubiquitin genes mentioned above. In contrast with the highly conserved nature of the proteinencoding portions of polyubiquitin genes, their promoters and introns have extensive sequence variability between paralogs and across organisms. However, all the polyubiquitin genes isolated from monocot and dicot plant species share similar structures, including a 5' UTR intron that significantly contributes to the strong expression capabilities of the polyubiquitin promoters [19,44-46].

In this study, we identified two novel polyubiquitin gene sequences (PvUbi1 and PvUbi2) from a switchgrass genomic library and characterized the native expression patterns of these genes. Additionally, reporter constructs were assembled containing the isolated 5 ' upstream regulatory regions of the coding sequences (i.e. PvUbi1 and PvUbi2 promoters) of these genes fused to the uidA coding region (GUS). These constructs were tested for transient and stable expression in a variety of plant species and tissues. Our results demonstrate the potential use of the PvUbi1 and PvUbi2 promoters in driving transgene expression in switchgrass, rice and tobacco. To the best of our knowledge, this is the first report characterizing native switchgrass promoter sequences for transgene expression.

\section{Results}

\section{Sequence analysis of PvUbi1 and PvUbi2}

Two polyubiquitin genes were cloned from a switchgrass fosmid library constructed by J. Hawkins and R. Percifield (unpublished results). These two genes were designated as ubiquitin 1 (PvUbi1) and 2 (PvUbi2) and are closely linked to each other within the genome (Figure 1). PvUbi1 consists of 607 bp containing cis-acting regulatory elements, a 5' untranslated region (UTR) containing a 93 bp non-coding exon and a 1291 bp intron, a 918 bp open reading frame (ORF) and a 191 bp 3' UTR. PvUbi2 consists of 692 bp containing cis-acting regulatory elements, a 97 bp 5' UTR, a 1072 bp intron, a 1146 bp ORF and a 183 bp 3' UTR. For PvUbi1, the 918 bp ORF encodes four tandem, head-to-tail repeats of $228 \mathrm{bp}$, commonly referred to as ubiquitin monomer repeats, with minimal sequence variation from one repeat to another. Similar results were found from sequence analysis of PvUbi2. However, instead of four repeats, the ORF contained five tandem head-to-tail repeats resulting in a coding region of $1146 \mathrm{bp}$. The ubiquitin monomers of PvUbi1 and PvUbi2 contained identical amino acid sequences compared to each other and several other plant species, including maize [18], Arabidopsis [47] and rice $[19,20]$. To test for promoter activity, the promoter candidate region of PvUbi1 that spans 607 bp of the 5' region upstream from the transcriptional initiation site, along with the $93 \mathrm{bp} 5$ ' UTR non-coding exon and the 1291 bp 5' UTR intron was cloned, resulting in a fragment of a total of $1991 \mathrm{bp}$. For PvUbi2, the isolated candidate promoter region was $1861 \mathrm{bp}$, including $692 \mathrm{bp}$ upstream of the transcriptional initiation site, the 5 ' UTR exon (97 bp) and the $1072 \mathrm{bp} 5$ ' UTR intron (Additional file 1 Figure $\mathrm{S} 1$ ). 


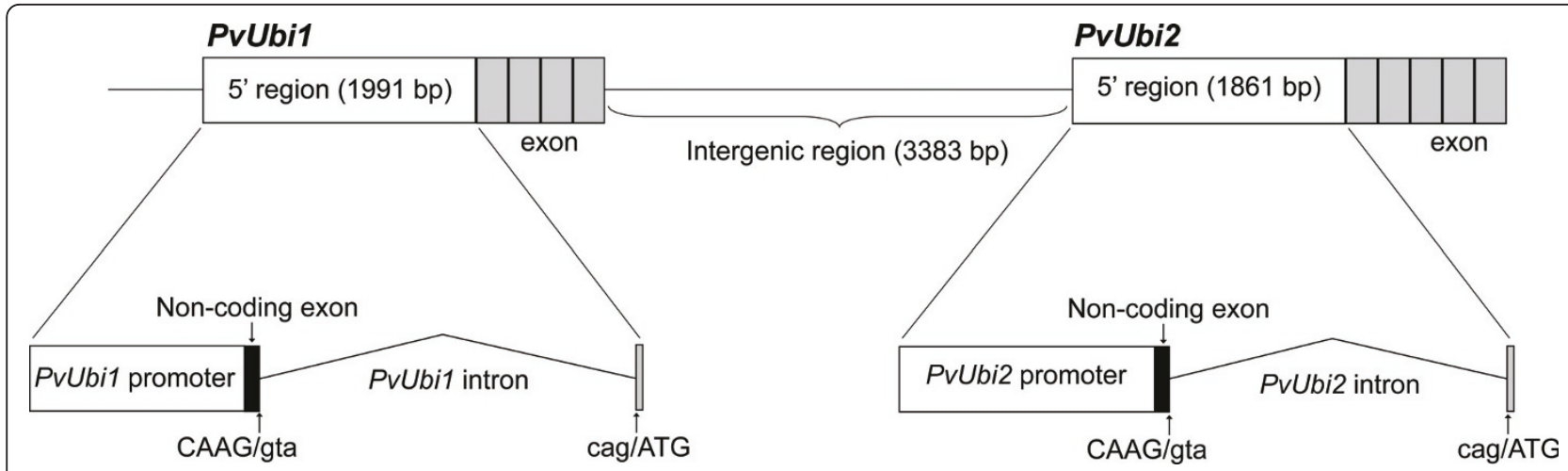

Figure 1 Map of the PvUbi1 and PvUbi2 genes within the contiguous 10.4 kb sequence of switchgrass DNA in Pv9G7B5. The light gray boxes represent the 228 bp ubiquitin monomer repeats of the translated exon. The black boxes represent the non-coding exons downstream of each TATA box for PVUbil and PVUbi2. The intron splices sites (CAAG/gta and cag/ATG) are shown.

By analysis of the genomic DNA in the selected promoter region, introns were identified immediately upstream of the ATG start codon in the PvUbi1 and PvUbi2 genes. These introns were identified based on the consensus sequences CAAG/gtac at the 5' splice site and cag/ATG at the 3' splice site (Additional file 1 Figure S2), which are identical for characterized polyubiquitin genes from plants [18-20,33-36,40,45]. To validate these intron splice sites and identify the transcriptional initiation site, switchgrass mRNA was subjected to RACE-PCR with primers specific for PvUbi1 or PvUbi2. For PvUbi1, results revealed a transcriptional initiation site at an adenine located 1384 bases upstream from the ATG translational initiation codon of the polyubiquitin gene. Subsequently, the transcriptional initiation site for PvUbi2 was identified at an adenine 1169 bp upstream from the translational initiation codon of the polyubiquitin gene. Using cDNA clones from the RACEPCR, along with expressed sequence tags (ESTs) from GenBank and Tobias et al. [48], the intron splice sites were confirmed, revealing 1291 and 1072 bp introns present in PvUbi1 and PvUbi2, respectively. The PvUbi1 and PvUbi2 introns exhibited limited homology (53\%), similar to the level of identity that has been reported when comparing different rice ubiquitin introns [20].

The PlantCARE Database [49] was queried for putative cis-element sequences within the candidate promoter regions of PvUbi1 and PvUbi2. Several motifs of putative functionality were identified for PvUbi1 and PvUbi2 (underlined in Additional file 1, Figure S2). For PvUbi1, these consisted of motifs involved in anaerobic induction (TGGTTT, positions -577 to -572), light-responsiveness (ATTAATTTTACA, positions -350 to -339; CACGTC, positions -589 to -584 ; $\mathrm{CC}(\mathrm{G} / \mathrm{A}) \mathrm{CCC}$, positions -221 to $216 ;-170$ to -165 ; and -54 to -49$)$, response to methyl jasmonate (MeJA) (CGTCA, positions -392 to -388 ; -77 to -73 ), low-temperature responsiveness (CCGAAA, positions -112 to -107 ), endosperm expression (GTCAT, positions -391 to -387), a MYB transcription factor binding site involved in drought-inducibility (TAACTG, positions -312 to -307 ), three CAAT boxes (positions -553 to -550 ; -538 to -535 ; and -458 to -455 ) and a TATA box (TATATAAA, positions -33 to -26 ). For PvUbi2, the identified motifs for putative cis-acting regulatory elements were those involved in meristem expression (GCCACT) and meristem specific activation (CCGTCC), anoxic-specific inducibility (CCCCCG), low-temperature responsiveness (CCGAAA), endosperm expression (GTCAT), light responsiveness (CC(G/A)CCC), two CAAT boxes and a TATA box (TAAATA, positions -32 to -27). However, it is important to indicate that these elements were determined from in silico data and remain to be functionally validated.

\section{Tissue expression profiles of PvUbi1 and PvUbi2}

A survey of switchgrass ESTs from the GenBank and Tobias et al. [48] databases revealed expression of PvUbi1 and PvUbi2 in all examined tissue types and growth stages: leaf, root, apex and stem, crown, callus, early floral buds and reproductive tissue, late flowering buds and seed development, and etiolated seedlings. To confirm these in silico data, specific primers were designed for PvUbi1 and PvUbi2 to perform quantitative reverse transcriptase-PCR ( $\mathrm{qRT}-\mathrm{PCR}$ ) in different switchgrass tissues (Figure 2). The qRT-PCR results confirm that both PvUbi1 and PvUbi2 are expressed in all tissues tested (leaf, flower, stem, root and callus). PvUbi2 showed higher levels of expression in all tissues except stem when compared to PvUbil.

\section{Transient and stable GUS expression regulated by the PvUbi1 and PvUbi2 promoters}

The capabilities of the PvUbi1 and PvUbi2 promoter candidate regions to drive transgene expression were evaluated through the construction of expression vectors 


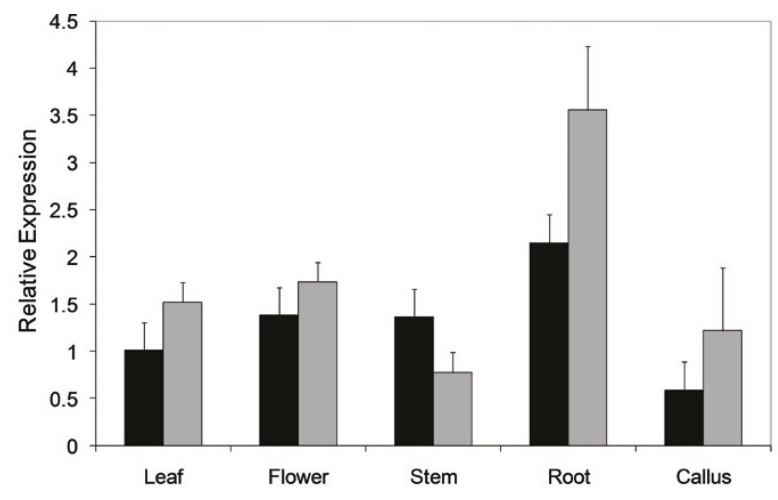

Figure 2 Expression analysis of switchgrass PvUbi1 (black) and PvUbi2 (gray) in a variety of switchgrass tissues using qRT-PCR. Relative quantification was performed using the standard curve method, and transcript accumulation of each gene was normalized to the quantity of an expressed switchgrass actin (PvAct) gene. Each bar represents the mean of three independent replicates with the standard errors of the noted mean.

(Additional file 1, Figure S2). All promoter variants were cloned upstream of the uidA coding region (GUS) to create promoter-GUS fusions. Vector constructs were transformed into switchgrass callus by particle bombardment, and transient expression was observed by GUS histochemical staining. All constructs showed GUS expression, verifying that the PvUbi1 and PvUbi2 promoters can be used successfully to drive transgene expression. In order to compare expression levels of the PvUbi1 and PvUbi2 promoters with those of other promoters, several plant promoters commonly used in monocot transformation were selected and cloned into the same identical vector (pHLucGWgus) to eliminate any discrepancies in expression levels as a result of vector backbone or vector size (Additional file 1, Figure S2).

In switchgrass and rice, the PvUbi1 and PvUbi2 promoters resulted in higher levels of GUS compared to the CaMV $35 S$ and $2 \times 35 S$ promoters (Figure 3 ). In switchgrass, the PvUbi1 and PvUbi2 promoters drove significantly higher GUS expression when compared side-byside with all other plant promoters (ZmUbi1, OsAct1, $2 \times 35 S$, CaMV 35S), with the PvUbi2 promoter reaching 2.1-fold higher levels than CaMV $35 S$ (Figure 3a). In rice, the PvUbi1 and PvUbi2 promoters resulted in 5.1- and 6.6-fold higher levels of expression when compared to CaMV 35S, respectively (Figure 3b). No enhancement of GUS activity was observed when three and nine amino acids of the PvUbi1 and PvUbi2 ubiquitin coding regions were fused to their respective promoters (Figure 3c and $3 \mathrm{~d}$ ). When comparing promoter expression levels between switchgrass and rice, the relative difference in absolute gene expression was 2.6- to 4.4-fold higher for rice. To further validate these transient assays, rice callus was bombarded with promoter- pHLucGWgus constructs and selected for stable transformation. When transgenic plants were grown to maturity, the PvUbi1 and PvUbi2 promoters produced GUS in leaves, stems and roots of mature rice plants, while no GUS was detected in the untransformed control (Figure 4).

The PvUbi1 and PvUbi2 promoter constructs were stably transformed into tobacco (cv. Xanthi) to evaluate expression levels of these promoters in a dicot expression system. Additional promoter variants containing three or nine amino acid fusions downstream of the PvUbi1 and PvUbi2 intron sequences were tested for the potential of increased transgene expression. Stably transformed $\mathrm{T}_{0}$ plants were randomly selected and grown to obtain seed for generation of $\mathrm{T}_{1}$ progeny. GUS from the PvUbi1, PvUbi1+3 and PvUbi1+9 promoter constructs could not be visually observed in $\mathrm{T}_{1}$ seedlings at 10 and 17 days after germination, so these promoter variants were not studied in subsequent experiments (data not shown). While the PvUbi2 promoter drove GUS expression in leaves, stems and roots at 10 and 17 days, the levels of expression were minimal when compared to $2 \times 35 S$ (Figure 5). However, the GUS expression dramatically increased with the PvUbi2 promoters containing an additional three and nine amino acid fusions from the ubiquitin coding region (PvUbi2+3 and PvUbi2 +9 ), exhibiting visibly detectable levels of GUS activity in the vascular tissue of leaves, stems, and roots. There was no visible enhancement of GUS from the PvUbi2 promoter constructs in tobacco following heat shock induction treatment for 60 minutes at $42^{\circ} \mathrm{C}$. However, heat shock induction was not experimentally tested within the native PvUbi1 or PvUbi2 genes in switchgrass.

Since the PvUbi2 promoters showed expression in seedlings, plants containing these promoter constructs were grown to maturity for further analysis. As shown in Figure 6, adult $\mathrm{T}_{1}$ plants showed staining of GUS in the pollen, pistil and leaves for the PvUbi2 promoter variants (PvUbi2, PvUbi2+3, PvUbi2+9). Similar to the data from young seedlings, the PvUbi2+3 and PvUbi2+9 promoters containing fusions showed the highest levels of expression. Expression of GUS in mature leaves under the control of $P v U b i 2$ promoter variants appeared to be specific to the vascular tissue. GUS expression driven by the PvUbi2+3 and PvUbi2+9 promoters in mature $T_{1}$ plants was observed to be lower than those in seedlings. GUS staining was consistently higher for the $2 \times 35 S$ promoter in both tobacco seedlings and mature adult $\mathrm{T}_{1}$ plants.

\section{Discussion}

This study demonstrates the isolation and characterization of two switchgrass polyubiquitin genes, PvUbi1 and 


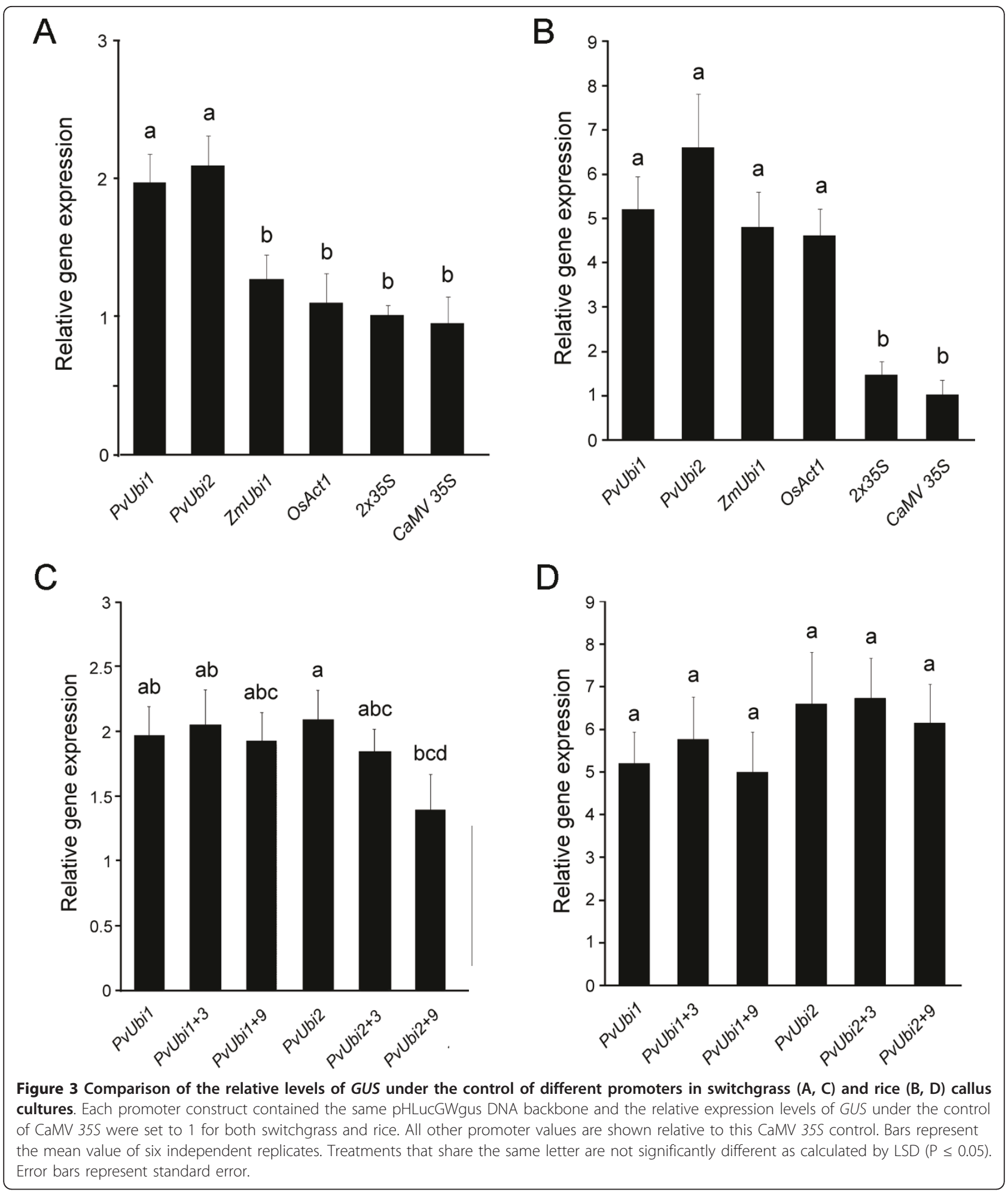

PvUbi2. The identical amino acid sequences of the ubiquitin monomer repeats in PvUbi1 and PvUbi2 further reveal the highly conserved nature of the polyubiquitin gene family across both monocot and dicot genes $[19,20,32,35,37,40]$. Both PvUbi1 and PvUbi2 displayed high levels of native expression in all tissue types analyzed, consistent with the broad function of ubiquitin in cell-cycle regulation [50], DNA-repair [51] and other processes required of all cell types [31]. Additional sequence data (unpublished) suggest that more than one 


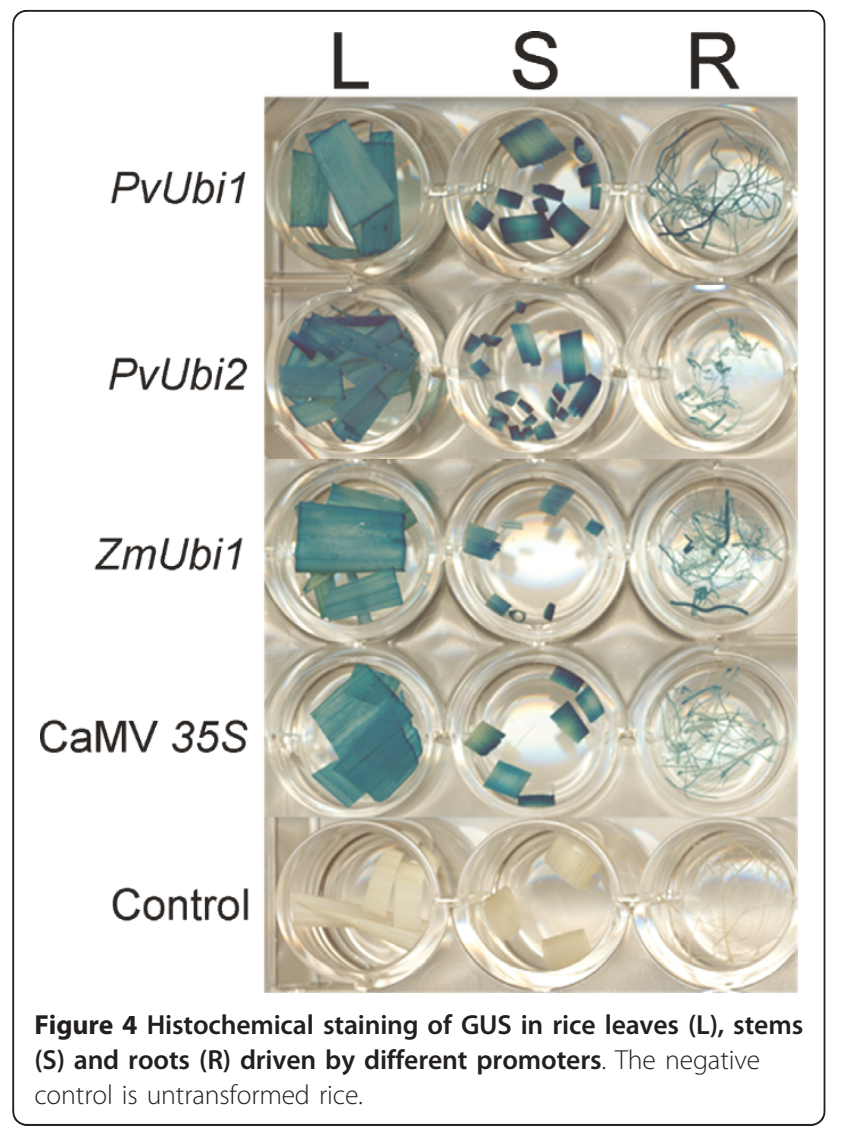

copy of polyubiquitin genes could exist in switchgrass containing 5' and 3' UTR regions identical to that of PvUbi1. These data are not unique to this study, as similar observations have been made in sugarcane, where 5' and 3' UTR sequences were identical for two individually isolated clones, while the number of ubiquitin monomer coding repeats varied [40]. Whether these findings are the results of different polyubiquitin genes present in the switchgrass genome or of post-transcriptional splicing and modification remains to be determined. In either case, the presence of multiple genes is consistent with the polyploidy nature of these species.

Intron-mediated enhancement of gene expression has been shown in a number of plant species [44,52], and multiple ubiquitin promoters have shown enhanced transgene expression when the intron is included in the promoter region $[19,44-46,53,54]$. Therefore, the intron regions (1291 bp for PvUbi1 and 1072 bp for PvUbi2) were retained with their respective $5^{\prime}$ upstream promoter candidate regions during vector construction. However, the regulatory elements of some monocot promoters retain high expression despite large deletions in the internal portions of the intron sequence, as long as efficient intron splicing is retained [16]. Therefore, future deletion analysis for the PvUbi1 and PvUbi2 promoter candidate regions could yield beneficial results,

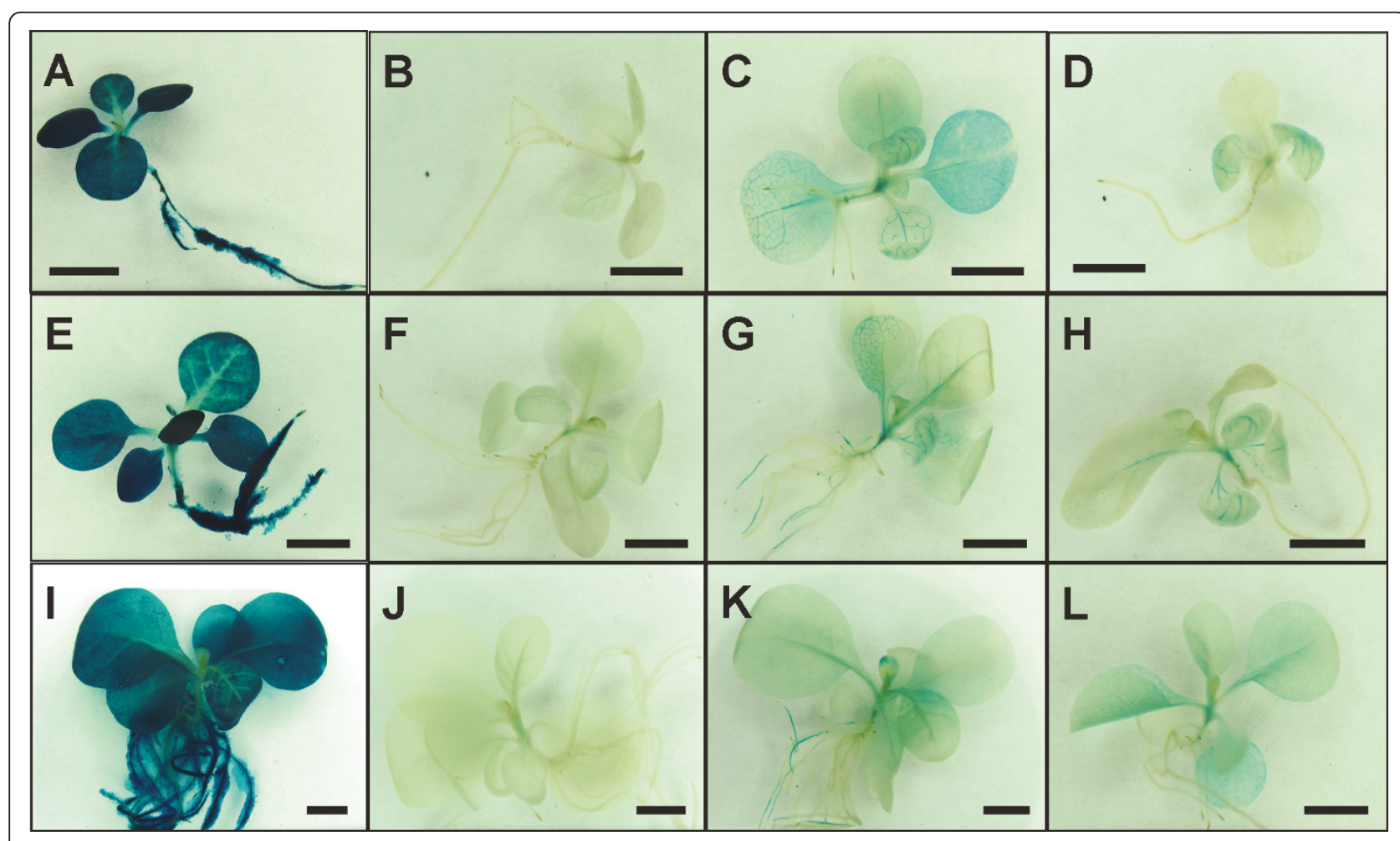

Figure 5 Histochemical staining of GUS in tobacco seedlings driven by the $2 \times 355(A, E, I), P v U b i 2(B, F, J), P v U b i 2+3(C, G, K)$ and PvUbi2+9 (D, H, L) promoters. Staining was performed at 10 days $(A, B, C, D)$ and 17 days $(E, F, G, H)$ post-germination. Heat shock was also performed at 17 days post-germination $(I, J, K, L)$. Each measurement bar represents $5 \mathrm{~mm}$. 


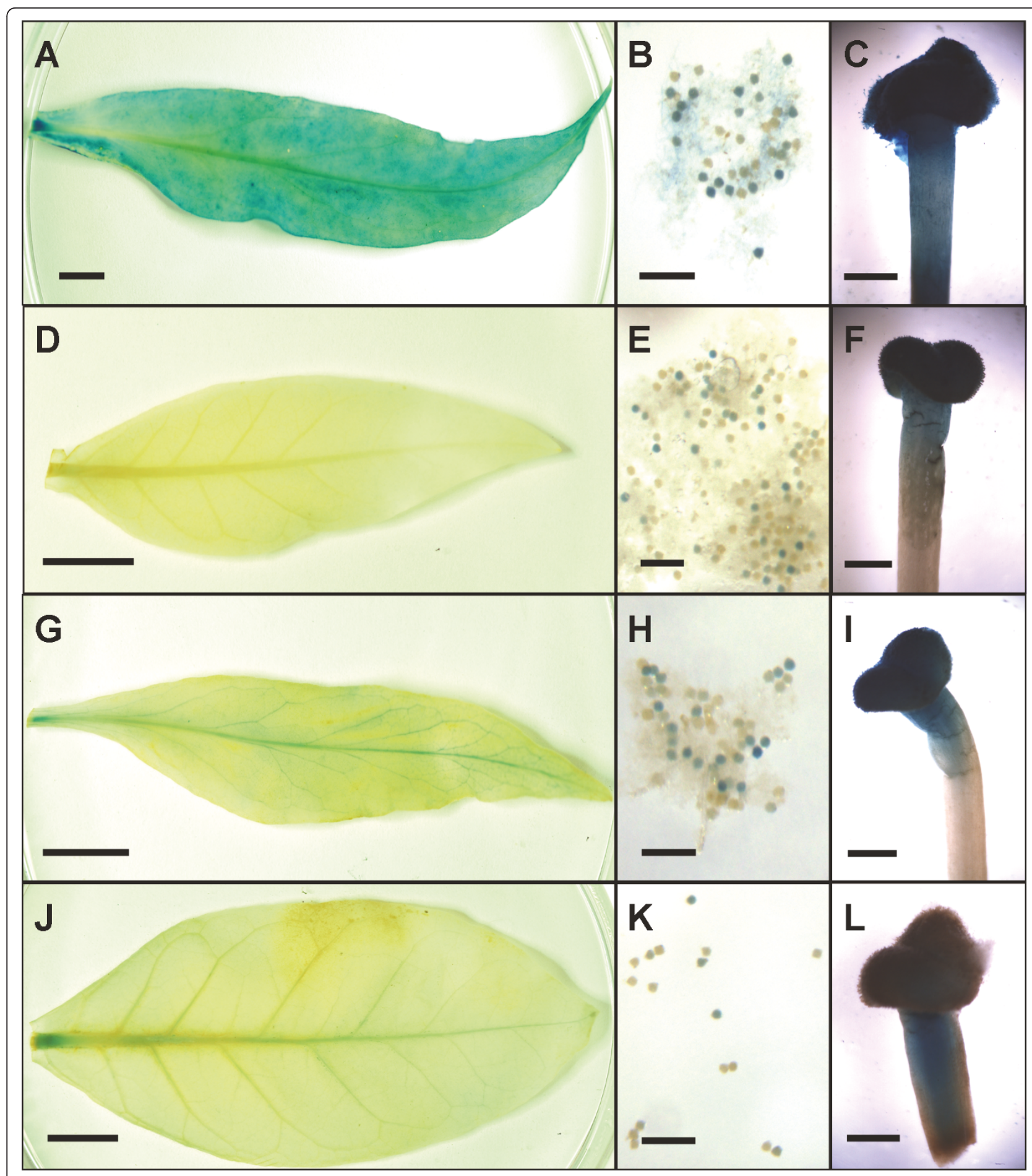

Figure 6 Histochemical staining of GUS in the leaves $(A, D, G, J)$, pollen $(B, E, H, K)$ and pistils $(C, F, I, L)$ of mature tobacco plants driven by the $2 \times 35 S$ (A, B, C), PvUbi2 (D, E, F), PvUbi2+3 (G, H, I) and PvUbi2+9 (J, K, L) promoters. Each measurement bar represents 1 $\mathrm{cm}$ (leaf), $0.2 \mathrm{~mm}$ (pollen) and $1 \mathrm{~mm}$ (pistil).

since the removal of internal ubiquitin intron sequences may have no detrimental effect [35].

The fusion of ubiquitin monomers to the $\mathrm{N}$ terminus of expressed proteins has been observed to lead to site- specific and highly efficient cleavage, resulting in free ubiquitin and free protein of interest in the cell [55]. These fusions are not only efficiently processed and cleaved by ubiquitin-specific proteases, but can also 
result in enhanced gene expression and protein accumulation $[19,56]$. Ubiquitin fusions have been applied for higher production of therapeutic recombinant proteins [57]. Additionally, plant vectors have been developed that employ ubiquitin fusions for the coexpression and cleaving of two proteins from a single transcript [58]. This approach has also been used for the enhancement of ubiquitin promoters in regulating transgene expression by the addition of an entire ubiquitin monomer or select amino acids from the $\mathrm{N}$ terminus of the polyubiquitin coding region downstream of the promoter region $[19,36,37,46]$. In this study, comparative expression levels of the PvUbi1 and PvUbi2 promoter candidate regions and their three (PvUbi $1+3$ and $P v U b i 2+3)$ or nine $(P v U b i 1+9$ and $P v U b i 2+9)$ amino acid ubiquitin fusion promoter variants revealed minimal changes in levels of GUS expression for switchgrass and rice. However, the three and nine amino acid fusions of the polyubiquitin coding region to the PvUbi2 promoter and intron drastically increased GUS staining in the vascular tissue of tobacco. While an additive effect has been observed when the first three or nine amino acids of the polyubiquitin coding region are fused to the $\mathrm{N}$-terminus of a transgene and coupled with a ubiquitin promoter [19], these enhancing effects were later attributed to the removal of a GUS 5' untranslated leader sequence [59]. Therefore, the enhancing effect of these fusions in tobacco is striking, since the majority of ubiquitin fusion enhancements have been demonstrated fusing an entire ubiquitin monomer to the transgene $[36,37,46,56,60]$. Fusion of the entire ubiquitin monomer was not tested in this study, but the high levels of expression in switchgrass and rice using the $P v U b i 1$ and $P v U b i 2$ promoters and introns without the ubiquitin monomer fusions demonstrates that these promoter and intron regions alone are highly useful for plant biotechnology applications in monocots. Likewise, the PvUbi2+3 and PvUbi2 +9 promoter variants could be useful for tissue-specific applications in tobacco and other dicots.

Data from transient biolistic bombardment assays can be highly variable in nature. Therefore, the rice and switchgrass transient expression assays were repeated in six independent replicates to increase the reliability of the resulting data. In addition, the pHLucGWgus vector used in these experiments contained the ZmUbi1 promoter and the firefly luciferase coding sequence ( $L U C$ ) in the vector backbone as an internal control to further improve the reliability and reproducibility of these transient expression data, as previously reported $[19,61,62]$. The methodology used for the bombardment assay in switchgrass and rice is the first plant transformation study of promoter expression using an internal LUC cassette control within the same vector backbone as the experimental promoter-GUS fusion cassette, as opposed to the standard use of co-transformation $[19,63,64]$.

The promoter-GUS fusions were observed to produce high levels of transgene expression in both switchgrass and rice. The comparisons of the expression levels of promoters PvUbi1 and PvUbi2 to other promoters are worth noting, since the CaMV $35 S$ and ZmUbi1 promoters are the two most commonly used in monocot transformation. The PvUbi1 and PvUbi2 promoters showed levels of expression higher than or equal to those of the ZmUbi1 and CaMV $35 S$ promoters. The PvUbi2 promoter should be the most effective for driving expression of transgenes in switchgrass; promoter PvUbi2 resulted in significantly higher expression levels than ZmUbi1 and CaMV 35S. In rice, the differences were even more striking, with the PvUbi2 promoter resulting in expression levels 6.6-fold higher than that of CaMV 35S, the most commonly used promoter in this species. The levels of gene expression detected in rice for the ZmUbi1, OsAct1 and CaMV $35 S$ promoters in this study reflect previous findings of high gene expression levels in maize [62]. The PvUbi1 and PvUbi2 promoters resulted in higher transgene expression in rice than in switchgrass, demonstrating the flexibility of these promoters in a monocot species other than switchgrass, and the potential for similar results in other monocots as well. The stable expression of GUS driven by PvUbi1 and PvUbi2 promoters in rice leaves, stems and roots also demonstrates the ubiquitous nature of these promoters in mature plants. These data suggest that the PvUbi1 and PvUbi2 promoters should be very effective for driving constitutive transgene expression in switchgrass, rice and potentially other monocots.

While expression of the PvUbi1 promoter was not observed in tobacco, the tissue-specific regulation of the PvUbi2 promoter and fusion variants could make these promoters advantageous for some applications that require expression limited to vascular or reproductive tissues. The decrease of GUS expression in adult leaf tissue is consistent with the findings that ubiquitin levels are higher in younger plant tissues $[47,65]$, although conflicting observations have been made with other ubiquitin promoters [66]. When the PvUbi2 promoter variants were tested for heat shock-induced transgene expression in tobacco, there was no effect observed on the level of GUS expression. However, it is worth noting that the $u b q 1-1$ promoter from tomato exhibited increased native transcript levels under heat shock conditions, but once fused to the GUS gene and stably expressed in tobacco, exhibited no response to heat shock induction [39], suggesting that the heat shock of ubiquitin promoters can yield different results when compared between native species and transgenic plant expression. In contrast, heat shock induction of the $Z m U$ Ubil promoter resulted in increased 
expression levels in native maize tissue as well as transgenic rice callus and protoplasts $[18,65,67]$, and sugarcane [40]. However, heat shock has been reported to have a variety of effects on polyubiquitin promoters including upregulation [18-20,33], down-regulation [38,68] and no change [34]. The heat shock element consensus sequence (CNNGAANNTTCNNG) reported by Pelham [69] was not found in the promoter regions of PvUbi1 or PvUbi2, and no heat shock elements were identified from the PlantCARE database queries [49]. However, the numerous putative cis-acting regulatory elements identified in the promoter regions of PvUbi1 and PvUbi2 could be functionally validated in future studies.

\section{Conclusion}

Because ubiquitin is a necessary component of all eukaryotic cells, polyubiquitin genes are prime candidates for the isolation of highly expressed constitutive promoters. We identified and characterized promoters from two polyubiquitin genes (PvUbi1 and PvUbi2) in switchgrass. Experiments using these promoters resulted in high levels of expression in switchgrass and rice that equaled or surpassed all of the commonly used plant promoters tested in this study (ZmUbi1, OsAct1, CaMV $35 S$ and 2x35S). In addition, stable transformation of tobacco with the PvUbi2 +3 and PvUbi2+9 promoter fusion variants showed expression in seedlings as well as the leaves, pistils and pollen of mature plants. These data suggest that the PvUbi1 and PvUbi2 promoters are valuable for genetic transformation studies and demonstrate the potential broad versatility of these promoters in monocot and dicot species.

\section{Methods}

\section{Construction and screening of a switchgrass fosmid} library

Panicum virgatum (cv. Alamo) leaf tissue was used to construct a fosmid library (unpublished data). In order to screen the library for polyubiquitin genes, $P$. virgatum EST sequence data (from JGI, Walnut Cove, CA) were aligned to genomic DNA sequences from rice and maize (NCBI). From these alignments, primers were designed to amplify fragments of 700-760 bp in size. Sequence specific primers (5'-TBACYGGMAAGACBATHACY-3', 5'-TCCTTYTGRATGTTRTARTC-3') were then used to screen the library. Fosmids identified to contain polyubiquitin genes were grown in 50-ml cultures containing $50 \mu \mathrm{l}$ of fosmid induction solution (Epicentre Biotechnologies) to increase copy number, as per manufacturer's protocol. Nuclear-free fosmid DNA was extracted using the Qiagen Large Construct Kit. Approximately $10 \mu \mathrm{g}$ of fosmid DNA [66 ng $\mathrm{\mu l}^{-1}$ ] was sheared to 2-10 kb using the Standard Hydroshear Shearing Assembly (Genomic Solutions) for 20 cycles at a speed code of 16 . Sheared fragments between 3 - $8 \mathrm{~kb}$ were excised and shotgun libraries were built as described [70]. Ten clones were randomly picked from the sub-clone library and digested with EcoRI to determine the average insert size as quality control. A total of 384 sub-clones were sequenced from both directions using ABI PRISM BigDye Chemistry (Applied Biosystems, Foster, CA) and run on an ABI 3730. The sequences were assembled using Phred/ Phrap and annotated in Apollo [71]. The cumulative data represent an approximate 13-fold coverage of each fosmid. Fosmid Pv9G7B5 contained two polyubiquitin genes in tandem and in the same orientation, both of which showed $\geq 99.5 \%$ sequence identity to switchgrass ESTs from callus, early floral development, late floral development, root, and stem tissues. Contig Pv9G7B5 was used for further isolation of switchgrass ubiquitin promoters.

\section{Sequence analysis}

Predictions were made for the location of the ubiquitin promoters and genes within the Pv9G7B5 fosmid using FGENESH [72] and GENSCAN [73] and further confirmed using blastn in GenBank and aligned with homologous ubiquitin sequences from other plant species in AlignX (Invitrogen, Carlsbad, CA). Based on these results, primers were designed to produce amplicons of PvUbi1 (1991 bp) and PvUbi2 (1861 bp) upstream of the predicted transcription start site (Additional file 1, Table S1). Identification of putative regulatory cis-elements within the promoter regions of PvUbil and PvUbi2 was performed using the PlantCARE database (http://bioinformatics.psb.ugent.be/webtools/plantcare/ html) [49].

\section{RACE-PCR}

The full-length cDNAs (including 5'UTRs, coding sequence and 3'UTRs) of the PvUbi1 and PvUbi2 genes were identified using the 5'RACE-PCR and 3'RACE$\mathrm{PCR}$, respectively, in the GeneRacer ${ }^{\mathrm{TM}}$ kit (Invitrogen, Carlsbad, CA, USA). Total RNA extractions from leaves of switchgrass $c v$. Alamo were performed using the TRI reagent (MRC, Cincinnati, $\mathrm{OH})$. Approximately $3 \mu \mathrm{g}$ of total RNA were used for reverse transcription to generate cDNA. To remove trace contamination of genomic DNA, RNA was treated with DNase I according to manufacturer's instructions (Promega, Madison, Wisconsin, USA). The resulting 5' and 3'UTRs of cDNA of both genes were amplified with the GeneRacer ${ }^{\mathrm{TM}}$ kit and cloned into $\mathrm{pCR}^{\mathbb{}} 8 / \mathrm{GW} / \mathrm{TOPO}^{\circledR}$ vector (Invitrogen) for sequence confirmation and analysis. The primers are listed in Additional file 1, Table S1.

\section{Quantitative reverse transcriptase PCR (qRT-PCR)}

Levels of PvUbi1 and PvUbi2 mRNA abundance were measured using quantitative reverse transcriptase PCR (qRT-PCR) in a variety of switchgrass tissues. Flower, 
leaf, stem and root tissues of three-month-old greenhouse-grown switchgrass (cv. Alamo), and callus generated from inflorescences of a switchgrass genotype (Alamo 2) [8] were used for RNA extraction. Total RNA was isolated using Tri-Reagent (Molecular Research Center, Cincinnati, $\mathrm{OH}$ ), and DNA contamination was removed with DNase treatment (Promega, Madison, WI) following the manufacturer's instructions. A switchgrass actin gene $(P v A c t)$ was used as an internal control. Specific primers to the corresponding genes were designed (Additional file 1, Table S1) that amplify a single product for each corresponding gene, as confirmed by the melting temperature of the amplicons and gel electrophoresis. Approximately $3 \mu \mathrm{g}$ of the total RNA from three independent experiments were synthesized into first strand cDNA using the High Capacity cDNA Reverse Transcription kit (Applied Biosystems, Foster City, CA) and qRT-PCR was conducted in triplicate using Power SYBR Green PCR master mix (Applied Biosystems) according to the manufacturer's protocol. Relative quantification was performed using the standard curve method, and transcript accumulation of each gene was normalized to the quantity of expressed switchgrass actin gene. For quality assurance purposes, only qRTPCR assays that resulted in standard curves with the following parameters were considered: 1) linear standard curve throughout the measured area, 2) standard curve slope between -3.5 and -3.2 , and 3) $R^{2}$ value above 0.99 .

\section{Expression vector construction}

All promoters (ZmUbi1, OsAct1, CaMV 35S, 2x35S, PvUbi1, PvUbi1+3, PvUbi1+9, PvUbi2, PvUbi2+3, PvUbi2 +9) were amplified with specific primer sets shown in Additional file 1 (Table S1) and cloned into pCR8/GW/ TOPO (Invitrogen, Carlsbad, CA). The PvUbi1 and PvUbi2 promoter variants were derived from the Pv9G7B5 contig mentioned above, the $Z m U$ Uil promoter from pAHC25 [74], the OsAct1 promoter from pCOR113 [75], the CaMV $35 S$ promoter from pBin-m-gfp5-ER [76], and the $2 \times 35 S$ promoter from pMDC32 [77]. DNA was confirmed by restriction enzyme digests for orientation, and clones containing the proper orientation were sequenceverified at the University of Tennessee Molecular Biology Resource Facility. These amplified promoter regions were introduced from the pCR8/GW/TOPO backbone into the binary vectors pGWB533 and pGWB535 [78] using the Gateway ${ }^{\circledR}$ LR Clonase ${ }^{\circledR}$ II enzyme mix (Invitrogen). The pGWB533 vector contains the Gateway ${ }^{\circledR}$ cassette upstream of the uidA coding region (GUS), resulting in promoter:GUS fusion constructs used for initial promoter analysis and tobacco transformations. For comparison of different promoters in switchgrass and rice, the pCR8/ GW/ZmUbi1 vector and the pGWB535 vector (containing the Gateway ${ }^{\circledR}$ cassette upstream of the firefly luciferase coding region $(L U C)$ ) were LR recombined and the resulting ZmUbi1:LUC cassette was cloned along with the Gateway ${ }^{\circledR}$ cassette upstream of GUS (cloned from pGWB533) and termed pHLucGWgus. The reporter gene cassettes were assembled, sequenced and annotated using Geneious v5.0.3 software [79]. Each unique promoter described above was LR recombined into the Gateway-compatible site of the pHLucGWgus vector upstream and in the correct frame for GUS protein synthesis and sequence verified.

\section{Plant materials and tissue culture}

Switchgrass $c v$. Alamo genotype ST1 was provided by Zeng-Yu Wang from the Noble Foundation [80]. Plants were maintained in the greenhouse by pruning tillers that matured beyond the boot stage [81] in a $42 \%$ sand, and 58\% Fafard 3B soil mix (Conrad Fafard, Inc., Agawama, MA) in 12-liter plastic pots. Growth conditions consisted of a 12-h light/12-h dark cycle under 400-watt halide lamps. The greenhouse temperatures ranged from $20-27^{\circ} \mathrm{C}$. Plants were watered daily, and fertilized weekly with $0.45 \mathrm{~kg}$ of Peters ${ }^{\circledR}$ Professional All Purpose Plant Food (St. Louis, MO) per 379 liters of water. The last culm node of switchgrass produced immature inflorescences at the E2R0 stages [81]. Culm nodes were identified as previously described by Alexandrova et al. [82]. The 6.5-cm explants were surface-sterilized with $70 \% \mathrm{EtOH}$ for 1 minute with gentle agitation. Explants were then placed in 15\% Clorox $^{\circledR}$ v/v supplemented with 0.01\% Tween-20 (Fisher Scientific, Pittsburgh, PA, USA) and gently agitated for 3 minutes. All tissues were then rinsed three times at 2-minute intervals. Sterilized internodes were cut in half longitudinally [82] and explants were placed cut-side down on solid Murashige and Skoog (MS) medium [83] supplemented with B5 vitamins, $5 \mu \mathrm{M}$ BAP and 3\% sucrose. Explants were incubated in a growth chamber at $25^{\circ} \mathrm{C}$, with cool-white fluorescent lighting (66-95 $\mu \mathrm{E} \mathrm{m}^{-2} \mathrm{~s}^{-1}$ ) 16-h light/8-h dark cycle for 14 days. After 14 days of culture, immature inflorescences were used to initiate embryogenic callus cultures. The inflorescences were dissected out and cut to obtain sections of rachis tissue measuring $1 \mathrm{~cm}$ in length. Inflorescence pieces were placed on solid N6E medium [84] and incubated at $27^{\circ} \mathrm{C}$ in the dark with subculturing at three-week intervals. After the second subculture, callus was separated from the inflorescences and arranged in a $5 \times 5$-grid pattern on plates. Friable embryogenic callus tissue was bulked for eight months with subcultures at three-week intervals and used in particle bombardment experiments.

Seeds of rice $c v$. Taipei 309 were provided by the USDA National Plant Germplasm System. Kernels from dehusked seeds were surface-sterilized in $70 \% \mathrm{EtOH}$ for 2 minutes at 100 RPM. Kernels were then transferred to a $60 \%$ Clorox $^{\circledR} \mathrm{v} / \mathrm{v}$ supplemented with $0.01 \%$ Tween-20, stirred for 30 minutes and rinsed three times with $\mathrm{H}_{2} \mathrm{O}$ 
for two minutes. Sterilized kernels were dried, arranged in a $5 \times 5$-grid on modified NB medium $(\mathrm{MNB})$ as per Chen et al. [85] and incubated in the dark at $27^{\circ} \mathrm{C}$. Prior to particle bombardment, rice callus was induced, selected, and maintained as previously described for 5 months with transfers at 3-week intervals [85]. All switchgrass and rice media were solidified with $2.5 \mathrm{~g} \mathrm{l}^{-1}$ Gelzan $^{\mathrm{TM}}$ (Caisson Laboratories, North Logan, UT, USA) and brought to $\mathrm{pH} 5.8$ prior to autoclaving. Cultures were sealed in Petri dishes with $3 \mathrm{M}$ Micropore ${ }^{\mathrm{TM}}$ tape (St. Paul, MN, USA).

DNA particle bombardment of switchgrass and rice callus Transient expression assays of Taipei 309 and ST1 embryogenic callus cultures were conducted following biolistic transformation using the Bio-Rad PDS-1000 (Bio-Rad Laboratories, Hercules, CA). The PDS-1000 was used for plasmid delivery with 7,584 $\mathrm{kPa}(1,100 \mathrm{psi})$ rupture disks, a microcarrier flight distance of $6 \mathrm{~cm}$ and a vacuum of 97 $\mathrm{kPa}(27 \mathrm{in}) \mathrm{Hg}[86,87]$, with all hardware and reagents produced by Bio-Rad. Microprojectile preparation essentially followed Trick et al. [88] with the DNA amount decreased from $625 \mathrm{ng}$ to $300 \mathrm{ng}$ per bombardment, and $10 \mathrm{mg}$ of $0.6 \mu \mathrm{m}$ diameter gold $(\mathrm{Au})$ particles used instead of $12 \mathrm{mg}$ of $1 \mu \mathrm{m}$ particles. Each bombardment consisted of a $10 \mu \mathrm{l}$ aliquot placed on the macrocarrier and allowed to dry completely. Switchgrass and rice callus cultures were incubated for $6 \mathrm{~h}$ prior to bombardment on N6 osmotic medium with $0.6 \mathrm{M}$ osmoticum (http://www.agron.iastate.edu/ ptf/protocol/Callus\%20bb.pdf), or 0.6 M NB osmotic medium [85], respectively. Each vector was used to bombard six replicate plates with 50 callus pieces per plate. To test the functionality of the promoter vectors and the validity of the bombardment assay, ten rice calli were selected from each of the first two replications and histochemically stained for observation of GUS. The five rice calli with the highest level of expression were selected and photographed (Additional file 1, Figure S3).

\section{Stable transformation of rice}

Stable transformations of rice were performed as described above for transient expression assays with three exceptions: three-month-old rice callus cultures were used and $150 \mathrm{ng}$ of the pHLucGWgus vectors (containing the PvUbi1, PvUbi1+3, PvUbi1+9, PvUbi2, PvUbi2+3, PvUbi2 +9, ZmUbi1, and CaMV 35 promoters) were used per bombardment. Rice callus cultures were incubated for $6 \mathrm{~h}$ pre- and $18 \mathrm{~h}$ post-bombardment on $0.6 \mathrm{MNB}$ osmotic medium [85]. Rice callus cultures were selected on MNBH50 as described [85] to ensure independent events were recovered. Positive transgenic calli were regenerated as described by Broothaerts et al. [89] on RGH6 medium solidified with Phytagel $\left(6 \mathrm{~g} \mathrm{l}^{-1}\right)$ without selection and resulting plantlets were rooted for four weeks on 1/2 MS medium supplemented with B5 vitamins and hygromycin B (50 mg l-1 $)$ solidified with $3 \mathrm{~g} \mathrm{l}^{-1}$ Gelzan $^{\mathrm{TM}}$ in Magenta ${ }^{\circledR}$ GA-7 Plant Culture vessels. Regeneration and rooting occurred under a 23-h light/1-hr dark photoperiod provided by cool-white fluorescent light $\left(66-95 \mu \mathrm{E} \mathrm{m}^{-2} \mathrm{~s}^{-1}\right)$ at $26^{\circ} \mathrm{C}$. Prior to being moved to the greenhouse, a root sample was harvested and GUS-stained for all transgenics, and an untransformed control was regenerated without selection. Plants were allowed to grow in the greenhouse for approximately two months prior to harvesting tissue for GUS staining of leaf and stem tissues.

\section{MUG and LUC assays}

Following bombardment, gene expression was analyzed using luciferase and MUG assays. Thirty-six hours postbombardment, 25 calli per replicate were ground in $50 \mu \mathrm{l}$ of $1 \times$ lysis buffer $(1 \times$ LB) [63]. For the first two replications, five calli were stained for GUS [90]. Upon lysing the cells, $350 \mu$ lof additional $1 \times$ LB were added to each sample. The cell lysates were centrifuged at $13,000 \mathrm{~g}$ for five minutes at ambient conditions; the tubes were then rotated $180^{\circ}$ and spun again. The soluble protein extracts produced from each sample were used for 4-methylumbelliferyl $\beta$-D-glucuronide (MUG) and luciferase assays $[63,64]$. For MUG assays, $50-\mu l$ of protein extract were added to $50 \mu \mathrm{l}$ of assay buffer ( $1 \mathrm{mM}$ MUG in $1 \times \mathrm{LB})$. Reactions were incubated at $37^{\circ} \mathrm{C}$ for 24 hours, and subsequently terminated with $100 \mu \mathrm{l}$ of stop buffer $(0.2 \mathrm{M}$ $\mathrm{Na}_{2} \mathrm{CO}_{3}$ in $\mathrm{H}_{2} \mathrm{O}$ ). Samples were read in duplicate with the BioTek ${ }^{B}$ Synergy 2 fluorometer (BioTek, Winooski, VT, USA) at an excitation wavelength of $360 / 40 \mathrm{~nm}$ and an emission wavelength of $460 / 40 \mathrm{~nm}$. The fluorometer was calibrated with 4-methyl umbelliferone (MU) standards in stop buffer. MUG results were expressed as micromole MU released hour ${ }^{-1}$. Luciferase activity was quantified twice for each replicate using $25 \mu \mathrm{l}$ of protein extract. For each sample reading, $25 \mu \mathrm{l}$ of sample extract in $1 \times$ LB buffer were diluted in $75 \mu \mathrm{l}$ of Glo-lysis buffer, mixed with $100 \mu \mathrm{l}$ of ONE-Glo ${ }^{\mathrm{TM}}$ Luciferase Assay buffer (Promega Corporation, Madison, WI, USA) and allowed to incubate at room temperature for 5 minutes. Non-specific GUS and luciferase activity was corrected, and normalization of the MUG data was accomplished using luciferase activity as previously described [62]. Each unique GUS cassette allowed the measurement of gene expression to be quantified. The strength of each promoter was reported relative to that of the CaMV $35 S$ control, normalized to 1 , to create a dimensionless value of promoter strength $[19,62]$.

\section{Agrobacterium-mediated transformation of tobacco}

The vectors to be tested were transformed into A. tumefaciens EHA105 as previously described [91]. EHA105 
cells were maintained in liquid YEP medium and all incubations were performed at $28^{\circ} \mathrm{C}$. Tobacco cv. Xanthi seeds were surface-sterilized, transformed, and regenerated using $50 \mathrm{mg} \mathrm{l}-1$ hygromycin for selection according to published methods [92].

\section{Histochemical staining}

Plant tissues were stained for GUS activity in microwell plates and placed at $37^{\circ} \mathrm{C}$ overnight as described [90]. For tobacco, intact tissue stains were made homogenous by vacuum infiltrating in solution for 30 minutes. For optimal visualization of stained tissues, chlorophyll was removed by repeatedly washing the tissue with a solution containing a 3:1 ratio of $\mathrm{EtOH}$ and acetic acid, ultimately storing tissue samples in 70\% EtOH for imaging. For rice tissues, GUS staining assays were completed using a modified GUS buffer [93] brought to $\mathrm{pH} 7$ [94], and chlorophyll was removed from the tissues as described by Cervera [95].

\section{Statistical analysis}

Data for relative expression of promoters using the MUG and LUC assays were subjected to Levene's test [96] to check for homogeneity of variance using the software package JMP ${ }^{\circledR}$ (Version 8.0.2 SAS Institute Inc., Cary, NC). When $\mathrm{p} \leq 0.05$, the data were considered to have unequal variances and were subjected to a square root transformation prior to ANOVA. Data sets with equal variances were subjected to ANOVA. If a significant difference was detected $(\mathrm{p} \leq 0.05)$ using ANOVA, the least significant difference test (LSD) was employed to analyze the data for significant differences between treatments within an experiment $(\mathrm{p}=0.05)$.

\section{GenBank accession numbers}

The PvUbi1 and PvUbi2 genes containing the promoters, 5 ' UTR exons and introns, polyubiquitin ORFs, and the 3' UTR regions have been deposited in GenBank (accession numbers HM209467 and HM209468, respectively).

\section{Additional material}

Additional file 1: Supplemental data.These data include sequences of the promoter candidate regions for PVUbil and PVUbi2, vector diagrams, representative images of the biolistic transformations and sequences of primers used in this study.

\footnotetext{
Acknowledgements

The authors gratefully acknowledge Dr. Zeng-Yu Wang (Samuel Roberts Noble Foundation) for providing the switchgrass ST1 plants for tissue culture and transformation. This work was supported by the Bioenergy Science Center. The BioEnergy Science Center is a U.S. Department of Energy Bioenergy Research Center supported by the Office of Biological and Environmental Research in the DOE Office of Science.
}

\section{Author details}

'Department of Plant Sciences, University of Tennessee, Knoxville, TN 37996, USA. ${ }^{2}$ Department of Crop and Soil Sciences, University of Georgia, Athens, GA 30602, USA. ${ }^{3}$ Department of Genetics, University of Georgia, Athens, GA 30602, USA. ${ }^{4}$ The BioEnergy Science Center, Oak Ridge National Laboratory, Oak Ridge, TN 37831-6026, USA.

\section{Authors' contributions}

DGJM designed and performed all experiments corresponding to the isolation and characterization of the individual gene elements and their respective promoters, the construction of vectors and the functional validation of the promoters in different species, analyzed the data and drafted the manuscript. ZRK designed and performed the promoter comparison assays in switchgrass and rice and assisted with revisions to the manuscript. WL designed and performed experiments of RACE-PCR and qRT$P C R$. BLJ performed stable transformation in tobacco and assisted with revisions to the manuscript. RJP and JSH constructed and screened the fosmid library for the presence of polyubiquitin genes. PRL participated in the design and construction of vectors. BJA and JNB participated in particle bombardment experiments. MM participated in experimental design, data analysis and assisted with revisions to the manuscript. JLB, WAP and CNS conceived and coordinated the study, and assisted with revisions to the manuscript. All authors read and approved the final version of this manuscript.

Received: 20 April 2011 Accepted: 11 July 2011 Published: 11 July 2011

\section{References}

1. Abramson M, Shoseyov O, Shani Z: Plant cell wall reconstruction toward improved lignocellulosic production and processability. Plant Science 2010, 178(2):61-72

2. Gressel J: Transgenics are imperative for biofuel crops. Plant Science 2008, 174(3):246-263.

3. Rubin EM: Genomics of cellulosic biofuels. Nature 2008, 454(7206):841-845.

4. Yuan JS, Tiller KH, Al-Ahmad H, Stewart NR, Stewart CN Jr: Plants to power: bioenergy to fuel the future. Trends in Plant Science 2008, 13(8):421-429.

5. Bouton $\mathrm{JH}$ : Molecular breeding of switchgrass for use as a biofuel crop. Current Opinion in Genetics \& Development 2007, 17(6):553-558.

6. McLaughlin SB, Walsh ME: Evaluating environmental consequences of producing herbaceous crops for bioenergy. Biomass and Bioenergy 1998, 14(4):317-324

7. Schmer MR, Vogel KP, Mitchell RB, Perrin RK: Net energy of cellulosic ethanol from switchgrass. Proceedings of the National Academy of Sciences 2008, 105(2):464-469.

8. Burris JN, Mann DGJ, Joyce BL, Stewart CN Jr: An improved tissue culture system for embryogenic callus production and plant regeneration in switchgrass (Panicum virgatum L.). BioEnergy Research 2009, 2(4):267-274.

9. Richards HA, Rudas VA, Sun H, McDaniel JK, Tomaszewski Z, Conger BV: Construction of a GFP-BAR plasmid and its use for switchgrass transformation. Plant Cell Reports 2001, 20(1):48-54.

10. Somleva MN, Snell KD, Beaulieu JJ, Peoples OP, Garrison BR, Patterson NA: Production of polyhydroxybutyrate in switchgrass, a value-added coproduct in an important lignocellulosic biomass crop. Plant Biotechnology Journal 2008, 6(7):663-678.

11. Somleva MN, Tomaszewski Z, Conger BV: Agrobacterium-mediated genetic transformation of switchgrass. Crop Sci 2002, 42(6):2080-2087.

12. Li R, Qu R: High throughput Agrobacterium-mediated switchgrass transformation. Biomass and Bioenergy 2011, 35(3):1046-1054.

13. Fu C, Mielenz JR, Xiao X, Ge Y, Hamilton CY, Rodriguez M, Chen F, Foston M, Ragauskas A, Bouton J, et al: Genetic manipulation of lignin reduces recalcitrance and improves ethanol production from switchgrass. Proceedings of the National Academy of Sciences 2011, 108(9):3803-3808.

14. Fu C, Xiao X, Xi Y, Ge Y, Chen F, Bouton J, Dixon RA, Wang Z-Y: Downregulation of cinnamyl alcohol dehydrogenase (CAD) leads to improved saccharification efficiency in switchgrass. BioEnergy Research 2011.

15. Saathoff AJ, Sarath G, Chow EK, Dien BS, Tobias CM: Downregulation of Cinnamyl-Alcohol Dehydrogenase in Switchgrass by RNA Silencing 
Results in Enhanced Glucose Release after Cellulase Treatment. PLOS ONE 2011, 6:(1):e16416.

16. He C, Lin Z, McElroy D, Wu R: Identification of a rice Actin 2 gene regulatory region for high-level expression of transgenes in monocots. Plant Biotechnology Journal 2009, 7(3):227-239.

17. McElroy D, Zhang W, Cao J, Wu R: Isolation of an efficient actin promoter for use in rice transformation. Plant Cell 1990, 2(2):163-171.

18. Christensen AH, Sharrock RA, Quail PH: Maize polyubiquitin genes: structure, thermal perturbation of expression and transcript splicing, and promoter activity following transfer to protoplasts by electroporation. Plant Molecular Biology 1992, 18(4):675-689.

19. Sivamani E, Qu R: Expression enhancement of a rice polyubiquitin gene promoter. Plant Molecular Biology 2006, 60(2):225-239.

20. Wang J, Jiang J, Oard JH: Structure, expression and promoter activity of two polyubiquitin genes from rice (Oryza sativa L.). Plant Science 2000, 156(2):201-211.

21. Mazarei M, Al-Ahmad H, Rudis MR, Stewart CN Jr: Protoplast isolation and transient gene expression in switchgrass, Panicum virgatum $\mathrm{L}$. Biotechnology Journal 2008, 3:354-359.

22. Sparkes IA, Runions J, Kearns A, Hawes C: Rapid, transient expression of fluorescent fusion proteins in tobacco plants and generation of stably transformed plants. Nat Protocols 2006, 1(4):2019-2025.

23. Li J-F, Park E, von Arnim A, Nebenfuhr A: The FAST technique: a simplified Agrobacterium-based transformation method for transient gene expression analysis in seedlings of Arabidopsis and other plant species. Plant Methods 2009, 5(1):6.

24. VanderGheynst JS, Guo HY, Simmons CW: Response surface studies that elucidate the role of infiltration conditions on Agrobacterium tumefaciens-mediated transient transgene expression in harvested switchgrass (Panicum virgatum). Biomass and Bioenergy 2008, 32(4):372-379.

25. Becker T, Templeman T, Viret J-F, Bogorad L: The cab-m7 gene: a lightinducible, mesophyll-specific gene of maize. Plant Molecular Biology 1992, 20:49-60.

26. Sullivan TD, Christensen A, Quail P: Isolation and characterization of a maize chlorophyll $a / b$ binding protein gene that produces high levels of mRNA in the dark. Molecular and General Genetics 1989, 215:431-440.

27. Lu J, Sivamani E, Li X, Qu R: Activity of the $5^{\prime}$ regulatory regions of the rice polyubiquitin rubi3 gene in transgenic rice plants as analyzed by both GUS and GFP reporter genes. Plant Cell Reports 2008, 27(10):1587-1600.

28. Peremarti A, Twyman R, Gómez-Galera S, Naqvi S, Farré G, Sabalza M, Miralpeix B, Dashevskaya S, Yuan D, Ramessar K, et al: Promoter diversity in multigene transformation. Plant Molecular Biology 2010, 73(4):363-378.

29. Saha P, Chakraborti D, Sarkar A, Dutta I, Basu D, Das S: Characterization of vascular-specific $R S s 1$ and rolC promoters for their utilization in engineering plants to develop resistance against hemipteran insect pests. Planta 2007, 226(2):429-442.

30. Sreekala C, Wu L, Gu K, Wang D, Tian D, Yin Z: Excision of a selectable marker in transgenic rice (Oryza sativa L.) using a chemically regulated Cre/loxP system. Plant Cell Reports 2005, 24(2):86-94.

31. Callis J, Vierstra RD: Ubiquitin and ubiquitin genes in higher plants. Oxford Surveys of Plant Molecular \& Cell Biology 1989, 6:1-30.

32. Callis J, Raasch JA, Vierstra RD: Ubiquitin extension proteins of Arabidopsis thaliana. Structure, localization, and expression of their promoters in transgenic tobacco. Journal of Biological Chemistry 1990, 265(21):12486-12493

33. Binet MN, Weil JH, Tessier LH: Structure and expression of sunflower ubiquitin genes. Plant Molecular Biology 1991, 17(3):395-407.

34. Kawalleck P, Somssich IE, Feldbrügge M, Hahlbrock K, Weisshaar B: Polyubiquitin gene expression and structural properties of the ubi4-2 gene in Petroselinum crispum. Plant Molecular Biology 1993, 21(4):673-684.

35. Genschik P, Marbach J, Uze M, Feuerman M, Plesse B, Fleck J: Structure and promoter activity of a stress and developmentally regulated polyubiquitin-encoding gene of Nicotiana tabacum. Gene 1994, 148(2):195-202.

36. Garbarino JE, Belknap WR: Isolation of a ubiquitin-ribosomal protein gene (ubi3) from potato and expression of its promoter in transgenic plants. Plant Molecular Biology 1994, 24(1):119-127.

37. Garbarino JE, Oosumi T, Belknap WR: Isolation of a polyubiquitin promoter and Its expression in transgenic potato plants. Plant Physiology 1995, 109(4):1371-1378.
38. Hoffman NE, Ko K, Milkowski D, Pichersky E: Isolation and characterization of tomato CDNA and genomic clones encoding the ubiquitin gene ubi3. Plant Molecular Biology 1991, 17(6):1189-1201.

39. Rollfink IK, Silber MV, Pfitzner UM: Characterization and expression of a heptaubiquitin gene from tomato. Gene 1998, 211(2):267-276.

40. Wei H, Wang M-L, Moore PH, Albert HH: Comparative expression analysis of two sugarcane polyubiquitin promoters and flanking sequences in transgenic plants. Journal of Plant Physiology 2003, 160(10):1241-1251.

41. Joung YH, Kamo K: Expression of a polyubiquitin promoter isolated from Gladiolus. Plant Cell Reports 2006, 25(10):1081-1088.

42. Chiera JM, Bouchard RA, Dorsey SL, Park E, Buenrostro-Nava MT, Ling PP, Finer JJ: Isolation of two highly active soybean (Glycine max (L.) Merr.) promoters and their characterization using a new automated image collection and analysis system. Plant Cell Reports 2007, 26(9):1501-1509.

43. Maekawa T, Kusakabe M, Shimoda Y, Sato S, Tabata S, Murooka Y, Hayashi M: Polyubiquitin promoter-based binary vectors for overexpression and gene silencing in Lotus japonicus. Molecular PlantMicrobe Interactions 2008, 21(4):375-382.

44. Mascarenhas D, Mettler IJ, Pierce DA, Lowe HW: Intron-mediated enhancement of heterologous gene expression in maize. Plant Molecular Biology 1990, 15(6):913-920.

45. Norris SR, Meyer SE, Callis J: The intron of Arabidopsis thaliana polyubiquitin genes is conserved in location and is a quantitative determinant of chimeric gene expression. Plant Molecular Biology 1993, 21(5):895-906

46. Plesse B, Criqui M-C, Durr A, Parmentier Y, Fleck J, Genschik P: Effects of the polyubiquitin gene Ubi.U4 leader intron and first ubiquitin monomer on reporter gene expression in Nicotiana tabacum. Plant Molecular Biology 2001, 45(6):655-667.

47. Burke T, Callis J, Vierstra RD: Characterization of a polyubiquitin gene from Arabidopsis thaliana. Molecular and General Genetics 1988, 213:435-443.

48. Tobias CM, Sarath G, Twigg P, Lindquist E, Pangilinan J, Penning BW, Barry K, McCann MC, Carpita NC, Lazo GR: Comparative genomics in switchgrass using 61,585 high-quality expressed sequence tags. The Plant Genome 2008, 1(2):111-124.

49. Lescot M, Dehais P, Thijs G, Marchal K, Moreau Y, Van de Peer Y, Rouze P, Rombauts S: PlantCARE, a database of plant cis-acting regulatory elements and a portal to tools for in silico analysis of promoter sequences. Nucleic Acids Research 2002, 30:325-327.

50. Ciechanover A, Finley D, Varshavsky A: Ubiquitin dependence of selective protein degradation demonstrated in the mammalian cell cycle mutant ts85. Cell 1984, 37:57-66.

51. Jentsch S, McGrath JP, Varshavsky A: The yeast DNA repair gene RAD6 encodes a ubiquitin-conjugating enzyme. Nature 1987, 329:131-134.

52. Rose $A B$, Beliakoff JA: Intron-mediated enhancement of gene expression independent of unique intron sequences and splicing. Plant Physiology 2000, 22:535-542.

53. Bianchi M, Crinelli R, Giacomini E, Carloni E, Magnani M: A potent enhancer element in the $5^{\prime}$-UTR intron is crucial for transcriptional regulation of the human ubiquitin C gene. Gene 2009, 448(1):88-101.

54. Lu J, Sivamani E, Azhakanandam K, Samadder P, Li X, Qu R: Gene expression enhancement mediated by the $5^{\prime}$ UTR intron of the rice rubi3 gene varied remarkably among tissues in transgenic rice plants. Molecular Genetics and Genomics 2008, 279(6):563-572.

55. Varshavsky A: Ubiquitin fusion technique and related methods. Methods in Enzymology 2005, 399:777-799.

56. Hondred D, Walker JM, Mathews DE, Vierstra RD: Use of ubiquitin fusions to augment protein expression in transgenic plants. Plant Physiology 1999, 119(2):713-724.

57. Mishra S, Yadav DK, Tuli R: Ubiquitin fusion enhances cholera toxin $B$ subunit expression in transgenic plants and the plant-expressed protein binds GM1 receptors more efficiently. Journal of Biotechnology 2006, 127(1):95-108.

58. Walker JM, Vierstra RD: A ubiquitin-based vector for the co-ordinated synthesis of multiple proteins in plants. Plant Biotechnology Journal 2007, 5(3):413-421.

59. Sivamani E, Starmer JD, Qu R: Sequence analysis of rice rubi3 promoter gene expression cassettes for improved transgene expression. Plant Science 2009, 177:549-556. 
60. Baker RT: Protein expression using ubiquitin fusion and cleavage. Current Opinion in Biotechnology 1996, 7(5):541-546.

61. Lepetit M, Ehling M, Gigot C, Hahne G: An internal standard improves the reliability of transient expression studies in plant protoplasts. Plant Cell Reports 1991, 10:401-405.

62. Schledzewski K, Mendel RR: Quantitative transient gene expression: comparison of the promoters for maize polyubiquitin1, rice actin1, maize-derived Emu and CaMV 355 in cells of barley, maize and tobacco. Transgenic Research 1994, 3:249-255.

63. Leckie F, Devoto A, Delorenzo G: Normalization of GUS by luciferase activity from the same cell extract reduces transformation variability. Biotechniques 1994, 17(1):52-53, 56-57.

64. Sivamani E, DeLong RK, Qu RD: Protamine-mediated DNA coating remarkably improves bombardment transformation efficiency in plant cells. Plant Cell Reports 2009, 28:213-221.

65. Cornejo M-J, Luth D, Blankenship KM, Anderson OD, Blechl AE: Activity of a maize ubiquitin promoter in transgenic rice. Plant Molecular Biology 1993, 23(3):567-581.

66. Basu C, Kausch AP, Luo H, Chandlee JM: Promoter analysis in transient assays using a GUS reporter gene construct in creeping bentgrass (Agrostis palustris). Journal of Plant Physiology 2003, 160(10):1233-1239.

67. Streatfield S, Magallanes-Lundback M, Beifuss K, Brooks C, Harkey R, Love R, Bray J, Howard J, Jilka J, Hood E: Analysis of the maize polyubiquitin-1 promoter heat shock elements and generation of promoter variants with modified expression characteristics. Transgenic Research 2004, 13(4):299-312.

68. Garbarino JE, Rockhold DR, Belknap WR: Expression of stress-responsive ubiquitin genes in potato tubers. Plant Molecular Biology 1992, 20:235-244.

69. Pelham HRH: A regulatory upstream promoter element in Drosophila Hsp70 heat-shock gene. Cell 1982, 30:517-528.

70. Dubcovsky J, Ramakrishna W, SanMiguel P, Busso CS, Yan L, Shiloff BA Bennetzen JL: Comparative sequence analysis of colinear barley and rice BACs. Plant Physiology 2001, 125:1342-1353.

71. Lewis SE, Searle SMJ, Harris N, Gibson M, lyer V, Richter J, Wiel C, Bayraktaroglu L, Birney E, Crosby MA, et al: Apollo: a sequence annotation editor. Genome Biology 2002, 3(12), RESEARCH0082.

72. Salamov AA, Solovyev W: Ab initio gene finding in Drosophila genomic DNA. Genome Research 2000, 10:516-522.

73. Burge C, Karlin S: Prediction of complete gene structures in human genomic DNA. Journal of Molecular Biology 1997, 268:78-94.

74. Christensen AH, Quail PH: Ubiquitin promoter-based vectors for highlevel expression of selectable and/or screenable marker genes in monocotyledonous plants. Transgenic Research 1996, 5:213-218.

75. McElroy $D$, Blowers $A D$, Jenes $B, W u R$ : Construction of expression vectors based on the rice actin 1 (Act1) $5^{\prime}$ region for use in monocot transformation. Molecular and General Genetics 1991, 231:150-160.

76. Haseloff J, Siemering KR, Prasher DC, Hodge S: Removal of a cryptic intron and subcellular localization of green fluorescent protein are required to mark transgenic Arabidopsis plants brightly. Proceedings of the National Academy of Sciences of the United States of America 1997, 94:2122-2127.

77. Curtis MD, Grossniklaus U: A Gateway cloning vector set for highthroughput functional analysis of genes in planta. Plant Physiology 2003, 133(2):462-469.

78. Nakagawa T, Suzuki T, Murata S, Nakamura S, Hino T, Maeo K, Tabata R, Kawai T, Tanaka K, Niwa Y, et al: Improved Gateway binary vectors: highperformance vectors for creation of fusion constructs in transgenic analysis of plants. Bioscience, Biotechnology, and Biochemistry 2007, 71(8):2095-2100.

79. Drummond AJ, Ashton B, Cheung M, Heled J, Kearse M, Moir R, StonesHavas S, Thierer T, Wilson A: Geneious v4.7. 2009.

80. Xi Y, Ge Y, Wang ZY: Genetic transformation of switchgrass. Biofuels 2009, 53-59.

81. Moore KJ, Moser LE, Vogel KP, Waller SS, Johnson BE, Pedersen JF: Describing and quantifying growth stages of perennial forage grasses. Agronomy Journal 1991, 83:1073-1077.

82. Alexandrova KS, Denchev PD, Conger BV: In vitro development of inflorescences from switchgrass nodal segments. Crop Science 1996, 36:175-178.

83. Murashige T, Skoog F: A revised medium for rapid growth and bio assays with tobacco tissue cultures. Physiol Plant 1962, 15(3):473-497.
84. Che P, Love TM, Frame BR, Wang K, Carriquiry AL, Howell SH: Gene expression patterns during somatic embryo development and germination in maize Hi II callus cultures. Plant Moleculuar Biology 2006, 62:1-14.

85. Chen L, Zhang S, Beachy RN, Fauquet CM: A protocol for consistent, largescale production of fertile transgenic rice plants. Plant Cell Reports 1998, 18(1):25-31.

86. Kikkert J: The Biolistic ${ }^{\ominus}$ PDS-1000/He device. Plant Cell, Tissue and Organ Culture 1993, 33(3):221-226

87. Sanford JC, Smith FD, Russell JA: Optimizing the biolistic process for different biological applications. Methods in Enzymology 1993, 217:483-509.

88. Trick HN, Dinkins RD, Santarem ER, Samoyolov RDV, Meurer C, Walker D, Parrott WA, Finer JJ, Collins GB: Recent advances in soybean transformation. Plant Tissue Culture and Biotechnology 1997, 3:9-26.

89. Broothaerts W, Mitchell HJ, Weir B, Kaines S, Smith LMA, Yang W, Mayer JE, Roa-Rodriguez C: Gene transfer to plants by diverse species of bacteria. Nature 2005, 433:629-633.

90. Jefferson RA, Kavanagh TA, Bevan MW: GUS fusions: beta-glucuronidase as a sensitive and versatile gene fusion marker in higher plants. EMBO Journal 1987, 6:3901-3907.

91. Hofgen R, Willmitzer L: Storage of competent cells for Agrobacterium transformation. Nucleic Acids Research 1988, 16(20):9877.

92. Horsch RB, Fry JE, Hoffmann NL, Eichholtz D, Rogers SG, Fraley RT: A simple and general method for transferring genes into plants. Science 1985, 227(4691):1229-1231.

93. Kosugi S, Ohashi Y, Nakajima K, Arai Y: An improved assay for $\beta$ glucuronidase in transformed cells: Methanol almost completely suppresses a putative endogenous $\beta$-glucuronidase activity. Plant Science 1990, 70(1):133-140.

94. Hodal L, Bochardt A, Nielsen JE, Mattsson O, Okkels FT: Detection, expression and specific elimination of endogenous $\beta$-glucuronidase activity in transgenic and non-transgenic plants. Plant Science 1992, 87(1):115-122.

95. Cervera M: Histochemical and fluorometric assays for uidA (GUS) gene detection. In Transgenic plants: Methods and protocols. Volume 286. Edited by: Peña L. New York, NY: Humana Press; 2005:203-213.

96. Levene $\mathrm{H}$ : Robust tests for the equality of variances. In Contributions to probability and statistics. Edited by: Olkin I. Palo Alto, CA: Stanford University Press; 1960:

doi:10.1186/1472-6750-11-74

Cite this article as: Mann et al: Switchgrass (Panicum virgatum L.) polyubiquitin gene (PvUbi1 and PvUbi2) promoters for use in plant transformation. BMC Biotechnology 2011 11:74.

\section{Submit your next manuscript to BioMed Central and take full advantage of:}

- Convenient online submission

- Thorough peer review

- No space constraints or color figure charges

- Immediate publication on acceptance

- Inclusion in PubMed, CAS, Scopus and Google Scholar

- Research which is freely available for redistribution

Submit your manuscript at www.biomedcentral.com/submit
C Biomed Central 